\title{
Geometric and Polynomial Approaches of Complex Systems and Control in Mathematics and Applied Sciences
}

\author{
Baltazar Aguirre-Hernández $\mathbb{D}^{1},{ }^{1}$ Jorge-Antonio López-Rentería ${ }^{(D)}{ }^{2}$ \\ Alejandro Armando Hossian, ${ }^{3}$ and Cutberto Romero-Meléndez ${ }^{1}$ \\ ${ }^{1}$ Universidad Autónoma Metropolitana, Mexico City, Mexico \\ ${ }^{2}$ CONACYT, TecNM, Instituto Tecnológico de Tijuana, B.C., Tijuana, Mexico \\ ${ }^{3}$ Universidad Tecnológica Nacional, Neuquén, Argentina \\ Correspondence should be addressed to Baltazar Aguirre-Hernández; bahe@xanum.uam.mx
}

Received 27 February 2020; Accepted 27 February 2020; Published 14 May 2020

Copyright (C) 2020 Baltazar Aguirre-Hernández et al. This is an open access article distributed under the Creative Commons Attribution License, which permits unrestricted use, distribution, and reproduction in any medium, provided the original work is properly cited.

Complex dynamical systems are present in many theoretical and practical domains of the science and engineering: physical processes, man-made systems, deterministic and stochastic control systems, distributed systems, and networks of leader-follower multiagent systems, between others.

The matrix approach of state space has long been the way to address many of the central problems of systems with control. In recent decades, novel methods and approaches for the study of systems, linear and nonlinear, with control are based on a geometrical approach whose objective is to reveal the properties of the geometric skeleton of the dynamic system. The geometric approach can convert a difficult nonlinear problem into a straight-forward linear one.

Geometric control theory and sub-Riemannian geometry are areas that play a very important role in complex dynamical systems, searching controllability, optimality, and stability for linear and nonlinear control systems, applying Lie theory techniques, Pontryagin maximum principle, and other geometric and algebraic techniques for robotic control, motion planning problems, complexity on path planning, neurobiological visual processing models, and digital image reconstruction.

On the contrary, the use of polynomial theory has been a useful tool to explain the classical and complex behavior of the solutions for a dynamical system, and it is largely exploited in fundamental problems such as controllability, stability, robustness, and other interesting applications in uncertain systems, nonlinear systems, time-delay systems, hybrid systems, and model predictive control.

In the paper "Poinacaré Map Approach to Global Dynamics of the Integrated Pest Management Prey-Predator Model," Zhenzhen Shi et al. studied, by means Poinacaré maps, the existence of periodic solutions of an integrated pest management prey-predator model with ratio-dependent and impulsive feedback control. The existence and stability of boundary order-one periodic solution is proved, and the authors give conditions for the existence and global stability of order $r-1$ periodic solution and for the existence of order $k$ periodic solution.

The paper "Availability Equivalence Analysis of a Repairable Bridge Network System," by Jaafar Alghazo et al., discusses availability equivalence factors of a repairable bridge network system where all components have constant failure and repair rates. The authors derive the availability of the original system and improved systems. Two types of availability equivalence factors of the system are obtained in order to compare different system designs. Numerical examples are included for illustrating the obtained results. The proposed models and analysis method are very useful in system engineering.

In the paper "On the Delay Interval in Which the Control Delay Systems are Stabilizable," Jiang Wei studies the stability of single delay systems and uses two-variable polynomials to derive some stability criteria. Besides, he uses these results to compute an interval for the delay where a 
two-dimensional control delay system is stabilizable by cases on their eigenvalues. Moreover, the general case is also deduced from its given results. Finally, an illustrative example is given.

The paper "Observer-Based Decentralized Tracking Control with Preview Action for a Class of Nonlinear Interconnected Systems," by Xiao Yu, investigates the problem of a decentralized output-feedback tracking control for a class of interconnected nonlinear systems, that is, the problem of asymptotic tracking control with preview action for a class of interconnected nonlinear systems. Such a control is converted into a regulation problem for the augmented systems comprising the tracking errors, the state observer, and the reference model. The problem formulation, consisting of the integral control action, the observerbased state feedback control action, and the desired trajectory preview action, is formulated in terms of LMIs by using Lyapunov theory. The resolution of these LMIs allows to calculate the decentralized observation and control gain matrices simultaneously. Furthermore, a numerical example is provided to show the performances of this control approach.

In the paper "Optimal Decay Rate Estimates of a Nonlinear Viscoelastic Kirchhoff Plate," B. Feng and M. Zahri establish optimal explicit and general energy decay results to the nonlinear viscoelastic Kirchhoff plate system by assuming minimal conditions on the relaxation function and a suitable proposing of energy and perturbed Lyapunov functions. This work is more general than previous results of Mustafa Zahri, and at last, some numerical illustrations are given to show the improvement of the optimal decay energy. The importance of studying the viscoelastic Kirchhoff plate equation is related to classical theory for beams/plates appearing from materials with viscoelastic structures, becoming this work novelty and interesting.

A study of the design of the nonlinear state feedback law for solving the nonlinear $H_{\infty}$ control problem is presented in the paper "Application of Sum of Squares Method in Nonlinear $H^{\infty}$ Control for Satellite Attitude Maneuvers" by Fanwei Meng et al. The authors propose a two-step iterative design method for solving nonlinear $H_{\infty}$ control. Firstly, they design a nonlinear state feedback of the gain array of the system, which is adjustable, by using the polynomial sum of squares method. Secondly, they solve the $L_{2}$ gain control, as the performance index. A satellite attitude maneuver control is taken as a nonlinear $H_{\infty}$ control example, and the optimization problem is solved by a graphical analytical method. Finally, simulation results are performed.

In the paper "Dynamic Analysis of Beddington-DeAngelis Predator-Prey System with Nonlinear Impulse Feedback Control," by Dezhao Li et al., a predator-prey model with a nonlinear pulse and functional Beddington-DeAngelis response is proposed. Firstly, the Poincaré map of the impulsive semidynamic system and discussion of its main properties including the monotonicity, differentiability, fixed point, and asymptote, was constructed. Some properties of the system are obtained by the Poincare map method, and the biological significance of the model is illustrated through numerical simulation. The results show that the pest population density can not only be controlled below the threshold under the state pulse feedback control but also avoid excessive application of pesticides and reduce some negative effects of pesticides.

The paper "Proportional PDC Design-Based Robust Stabilization and Tracking Control Strategies for Uncertain and Disturbed T-S Model," by Chekib Ghorbel et al., presents a proportional parallel-distributed compensation (PPDC) design for robust stabilization and tracking control of the nonlinear dynamic system, which is described by uncertain and perturbed Takagi and Sugeno (T-S) fuzzy model. The investigated topic is interesting, and the mathematic explanation of the proposed solutions is also nice. Furthermore, simulation results are used to illustrate the effectiveness of the proposed algorithm. Its interest is to reduce the number of adjustable parameters in the normal PDC one. Also, an inverted pendulum system was considered to show the effectiveness of the designed fuzzy controllers.

\section{Conflicts of Interest}

The editors declare that they have no conflicts of interest regarding the publication of this special issue.

\section{Acknowledgments}

The guest editorial team thanks the authors and the reviewers of the papers of this special issue who made this special issue viable.

Baltazar Aguirre-Hernández Jorge-Antonio López-Rentería Alejandro Armando Hossian Cutberto Romero-Meléndez 